\title{
Statistical analysis of the influence of tooth geometry in the performance of a harmonic drive
}

\author{
Dennis León ${ }^{1}$, Nelson Arzola ${ }^{2 *}$, Andrés Tovar ${ }^{3}$ \\ ${ }^{1,2}$ Department of Mechanical and Mechatronic Engineering \\ National University of Colombia \\ Carrera 30 45-03, Office 453-403, Bogotá, Colombia \\ ${ }^{3}$ Department of Mechanical Engineering \\ Purdue School of Engineering and Technology \\ Indiana University-Purdue University Indianapolis \\ 723 West Michigan Street, SL 260N, Indianapolis, Indiana 46202-5132, USA \\ *Tel. (57)3615000, Fax. (57)3165333, Email: narzola@unal.edu.co
}

\begin{abstract}
The objective of this research is to determine the influence of gear tooth geometrical variations on the performance of a double wave harmonic drive through stochastic analysis. This work incorporates state of the art analytical and numerical models to evaluate kinematic error, load capacity, bending fatigue strength, and pitting life. The geometric variables considered in this study include gear modulus, pressure angle, and tooth correction factor. The stochastic analysis follows a three-levels, full factorial design of experiments. Non-linear dynamic simulation is accompanied by finite-element analysis (FEA) to estimate contact and bending stresses. Largest bending fatigue strength is also determined. Results demonstrate that gear modulus is the geometric parameter with prevalent influence on the kinematic error, and pitting life is rather high for all geometric variables considered.
\end{abstract}

Keywords: Harmonic drive; analysis of variance; kinematic error; fatigue failure; design for strength

\section{Nomenclature}

$\alpha \quad$ Angle in coordinate system attached to the wave generator (rad)

$\beta \quad$ Angle in a reference coordinate system (rad)

$\delta_{a} \quad$ Setup gap of the flexible spline on the track of the wave generator (mm)

$\xi \quad$ Correction factor

$\xi_{1} \quad$ Correction factor for the circular spline

$\xi_{2} \quad$ Correction factor for the flexible spline

$\theta_{e} \quad$ Kinematic transmission error (rad)

$\theta_{i n} \quad$ Angular position of the input shaft (rad)

$\theta_{\text {out }} \quad$ Angular position of the output shaft (rad)

$\sigma_{e} \quad$ Corrected endurance limit for the flexible spline material (MPa)

$\sigma_{u t} \quad$ Ultimate tension stress (MPa)

$\sigma_{V M a} \quad$ Equivalent amplitude of the von Misses stress (MPa)

$\sigma_{V M m} \quad$ Equivalent mean of the von Misses stress (MPa)

$\sigma_{z} \quad$ Peak normal stress on the teeth of the flexible spline (MPa)

$\vartheta, \gamma \quad$ Pitting strength empirical parameters

$\Phi \quad$ Pressure angle $\left({ }^{\circ}\right)$

a Larger semi-axis of the wave generator ( $\mathrm{mm})$

$a^{\prime} \quad$ Larger pitch semi-axis (mm)

$b \quad$ Smaller semi-axis of the waver generator (mm)

$b^{\prime} \quad$ Smaller pitch semi-axis (mm)

$d_{e} \quad$ External diameter of the flexible spline (mm)

$h_{f} \quad$ Thickness of the flexible spline (mm)

$i \quad$ Transmission ratio

$k \quad$ Iteration time

m Modulus (mm)

This is the author's manuscript of the article published in final edited form as:

León, D., Arzola, N., \& Tovar, A. (2015). Statistical analysis of the influence of tooth geometry in the performance of a harmonic drive. Journal of the Brazilian Society of Mechanical Sciences and Engineering, 37(2), 723-735.

http://dx.doi.org/10.1007/s40430-014-0197-0 


$\begin{array}{cl}m_{1} & \text { Material elastic properties of the circular spline }\left(\mathrm{MPa}^{-1}\right) \\ m_{2} & \text { Material elastic properties of the flexible spline }\left(\mathrm{MPa}^{-1}\right) \\ r & \text { Pitch of the rigid spline (mm) } \\ t & \text { Time (s) } \\ D_{i} & \text { Internal diameter of the rigid spline (mm) } \\ \boldsymbol{F} & \text { Vector of internal nodal forces (N) } \\ \boldsymbol{K} & \text { Jacobian matrix } \\ N_{p} & \text { Number of cycles for pitting failure (cycles) } \\ P & \text { Reference point on the rigid spline } \\ \boldsymbol{R} & \text { Vector of external nodal forces (N) } \\ S_{f} & \text { Fatigue safety factor } \\ \boldsymbol{U} & \text { Vector of nodal displacements (mm) } \\ Z_{1} & \text { Number of teeth of the rigid spline } \\ Z_{2} & \text { Number of teeth of the flexible spline }\end{array}$

\section{Introduction}

A harmonic drive (HD) is compact coaxial gear mechanism characterized by high reduction ratios (up to 300:1), no backlash, and a few number of components, which makes it popular in robotics and automotive applications [1, 2]. A typical HD consists typically consists of three main coaxial components: a circular rigid spline, a flexible spline, and a wave generator. The wave generator, which is usually an elliptic cam, runs insight the flexible spline and deforms it to engage the outer circular rigid spline along the major elliptic axis. When the wave generator deforms the flexible spline in such a way that there are two points of contact with the rigid spline, the mechanism receives the name of double wave HD.

HDs offer several advantages with respect to conventional spur gear transmissions, including better mass-to-torque ratio and smaller overall dimensions than a spur gear equivalent. Since HDs have a flexible component and several teeth engaging simultaneously during the transmission, there is damping factor that is four to five times better than the spur gear counterparts [3]. The flexible teeth gearing results in theoretically zero backlash. Despite manufacturing challenges due to small moduli (from $0.10 \mathrm{~mm}$ to $2.00 \mathrm{~mm}$ ), HDs have been rapidly growing in popularity.

Numerical and analytic models have been developed to quantify life according to failure criteria and transmission conditions [4]. The solution of the harmonic flexural wave transmission kinematics is generally approached in two different ways. The first approach makes use of mathematical models of different transmission, incorporating structural and manufacturing parameters and results of experimental tests [5]. Lagrange equations are sometimes used in this approach [6]. The second approach is based on physical transmission models, which are tested for fatigue failure to determine performance and validate geometric parameters. This approach makes use of an analytic model that defines a harmonic curve trajectory. This model is based on the displacements of the wave generator and the flexible spline ideal displacement relation.

Longer fatigue lifetime is usually obtained for transmission ratios higher than 120:1. Fatigue lifetime considerably decreases for transmission ratios lower than $80: 1$. This is because the radial deformation that is required for a proper gearing action increases when the number of teeth is decreased. However, if geometrical and material parameters are carefully selected to maintain low deformation, the transmission ratio should not be a performance limiting factor.

The load conditions on the HD components can be stated in terms of energy conservation [7] or stated in term of variable relations [6]. The stress analysis makes use of analytical models that describe the flexible gear stress state as a function of the radial strain and geometric parameters [8]. Due to the complexity of the teethed component geometry, this is usually simplified so the approximated model captures the main features of a specific geometric profile and reduces the effect of some geometric variables [9]. One approach is to define a flexible line and model the HD structure according to the Kirchhoff-Love theory for plates and cylindrical shells [5]. Iterative methods are sometimes required in order to achieve a reliable approximation when using finite element analysis (FEA) to simulate dynamic loading conditions, nonlinear material, and varying boundary conditions due to contact [4]. The HD hysteresis can be obtained from torque analysis as a function of rotation using elastic deformation of the output shaft. The corresponding kinematic error can be obtained from a time history of the input and output shaft rotation. One procedure is to obtain a polar coordinate system from which the outer radius of the flexible gear is obtained. This radius is modified according 
to the harmonic and the angular positions. Once the input and output angles are determined, it is possible to obtain the kinematic transmission error $[7,10]$.

HD technology is in stage of development and innovation and designers strive to determine the influence of the design parameters on the HD performance using theoretical and experimental methods [4, 5, 9, 11]. The traditional approach is the use of sensitivity analysis, which refers to the variation of a generic performance function to small variations of parameters about a particular configuration [12]. This approach provides relevant information, but the results greatly depend on the selection of the particular configuration. In contrast to this traditional sensitivity analysis, we propose the use of global sensitivity analysis using a three-level full-factorial design of experiments [13]. This method is reliable though computationally demanding. A full-factorial design of experiments considers all possible combinations of design parameters $\left(n_{P}\right)$ and levels $\left(n_{L}\right)$ so the number of experiments is $n_{L}^{n_{P}}$. Two or three levels are typically employed, but the number of sample experiments may result very high; however, the reduction on the number of experiments decreases the computational cost, but also decreases the accuracy.

Therefore, the objective of this work is to provide HD design insights with the use of three-level full factorial experimental design considering the geometric variables with the most relevant influence on the gears' kinematics and the stress levels in a nonlinear dynamic finite-element model of a double wave HD. The design parameters include gear modulus, pressure angle, and tooth correction. The height of the teeth is also analyzed and corrected to achieve a satisfactory gear mesh. Performance parameters include kinematic error, load capacity, bending fatigue strength, and surface fatigue strength (pitting). The nonlinear dynamic model developed in our work provides the required a level of accuracy not commonly found in most studies currently available. In addition, the statistical analysis based on full factorial experimental design has not been previously reported and it is also considered a contribution of this work. The results of the statistical analysis are presented in the Appendix and analyzed with detail in the following sections.

\subsection{CAD model and simulation parameters}

\section{Materials and Methods}

Harmonic drives are composed of three main concentric components: an internal rigid spline, an external flexible spline, and a wave generator (Figure 1). The wave generator deflects the flexible external spline that meshes with the slightly larger, rigid, stationary internal circular spline. The wave generator, usually an ellipsoidal cam, is attached to the input shaft and acts as an efficient stress converter [11]. The flexible spline serves as the output, rotating in a direction opposite to one of the input shaft. Both splines have the same circular pitch, but the flexible spline typically has two teeth less than the rigid spline [14]. While the wave generator rotates, the contact line between the splines moves with the input shaft. For every $180^{\circ}$ of the input shaft, the flexible spline rotates one tooth in the opposite direction.
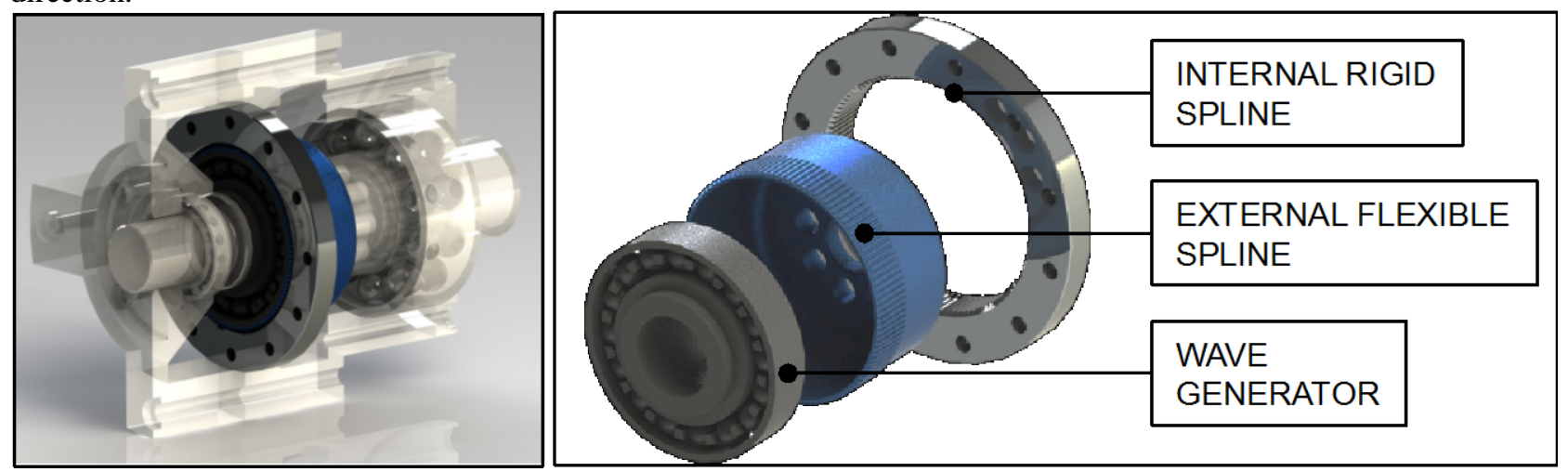

Figure 1: Harmonic drive assembly (left) and three main concentric components (right): an internal rigid spline, an external flexible spline, and a wave generator.

The splines in a HD are designed following a similar procedure than the one used to design planetary transmissions. First, the modulus is defined and then the number of teeth is determined. The number of teeth depends on the required number of waves. The transmission ratio $i$ is defined as [15]:

$$
i=\frac{Z_{1}}{Z_{1}-Z_{2}}
$$


where $Z_{1}$ and $Z_{2}$ are the number of teeth of the rigid and flexible splines, respectively. For example, two waves in the flexible spline can be generated for $Z_{1}=115$ and $Z_{2}=113$, case in which $i=57.5$.

The geometry configuration imposes that the diametral pitch of the rigid spline should be equal to the larger semi-axis diametral pitch of the flexible spline. Also, the internal perimeter of the flexible spline should be equal to the external elliptic perimeter of the wave generator. This condition is satisfied for [9]:

$$
d_{e}=D_{i}=\frac{1}{2} m\left(Z_{1}+Z_{2}\right),
$$

where $d_{e}$ is the external diameter of the flexible spline before setup (mm), $D_{i}$ is the internal diameter of the rigid spline before setup (mm), and $m$ is the modulus. The elliptic shape of the wave generator imposes another geometric constraint, in which the perimeter of the flexible spline should be maintained after setup. The perimeter of an ellipse can be determined from Ramanujan's equation

$$
\frac{1}{\pi} m Z_{2}=3\left(a^{\prime}+b^{\prime}\right)-\sqrt{10 a^{\prime} b^{\prime}+3\left(a^{\prime 2}+b^{\prime 2}\right)},
$$

where $a^{\prime}$ is the larger pitch semi-axis (mm), and $b^{\prime}$ is the smaller pitch semi-axis (mm) defined in this work by

$$
\begin{aligned}
& a^{\prime}=a+h_{f}+1.25 m+\delta_{a} \\
& b^{\prime}=b+h_{f}+1.25 m+\delta_{a},
\end{aligned}
$$

where $a$ is the larger semi-axis of the wave generator $(\mathrm{mm}), b$ is the smaller semi-axis of the waver generator (mm), $h_{f}$ is the thickness of the flexible spline $(\mathrm{mm})$, and $\delta_{a}$ is the setup gap of the flexible spline on the track of the wave generator (mm). In this model, all HD components have a constant thickness in the axial direction. The analytic definition of the involute is used to numerically generate the points that define the teeth profiles with the required precision. Tooth fillet radius corresponds to one sixth of the modulus.

The model meshing and finite element analysis are performed using CAE software SolidWorks and CosmosWork developed by Dassault Systèmes SolidWorks Corporation [16]. The bulk of each component is discretized using isoparametric four-noded triangular elements. The mesh size of the surfaces in contact is refined and its order increased to ten-noded elements that include angular and midsize nodes. The domains in this work have complex geometries where elements with square corners do not satisfied strictly the discretization. In this case, isoparametric elements are used. The mesh discretization and refinement are parameterized with respect to the gear modulus and contains about 23,000 triangular elements and 13,000 nodes. The finite element analysis is done under plane stress (Section 2.3).

\subsection{Kinematic error analysis}

The kinematic error $\theta_{e}$ of a harmonic drive is defined as [7]:

$$
\theta_{e}=\frac{\theta_{\text {in }}}{i}-\theta_{\text {out }}
$$

where $\theta_{\text {in }}$ and $\theta_{\text {out }}$ are the angular positions of the input and output shafts, respectively. Two models are considered to determine the kinematic transmission error: analytical kinematic model analysis and finite element-based numerical kinematic model. The analytical model describes the theoretical motion of the transmission and gives a kinematic approximation of the characteristic positions. This result is used as a reference to compare the deformations obtained from the finite element model. The analytic model describes the trajectory of a point on the flexible spline using geometric relations between the wave generator and the rigid spline. This model defines a straight joining the center of the wave generator and a point $P$ on the circular spline perimeter and two associated angles: $\alpha$ and $\beta$. Angle $\alpha$ is the rotation of the straight line measured in a coordinate system attached to the wave generator, and $\beta$ is the rotation of the straight line measured in a reference coordinate system [10]. In a generalized reference system, the displacement of $P$ can be described by the deviation between $\alpha$ and $\beta$. Since the ratio $i$ is constant, a correction needs to be applied. The ellipse coordinates $(x, y)$ in the moving coordinate system attached to the wave generator can be expressed in fixed coordinate system $\left(X_{0}, Y_{0}\right)$ as [17]:

$$
\begin{gathered}
X_{0}=-r \sin (\beta)=-\frac{y}{\cos (\alpha(i+1))} \sin (i \alpha) \\
Y_{0}=r \sin (\beta)=-\frac{y}{\cos (\alpha(i+1))} \cos (i \alpha)
\end{gathered}
$$

where $r$ is the pitch of the rigid spline (mm). Then, the position (velocity and acceleration) of $P$ can be defined, as well as the position (velocity and acceleration) of the flexible spline with respect to the wave generator. 
The finite element-based model can simulate the HD kinematics using the angular velocity of the wave generator, input torque, elastic properties, and boundary conditions. This numerical model can be obtained for splines components with and without teeth [14]. For a given time interval $t+\Delta t$, the equilibrium equation between external and internal forces is given by [18]:

$$
{ }^{t+\Delta t} \boldsymbol{R}-{ }^{t+\Delta t} \boldsymbol{F}=\mathbf{0},
$$

where $\boldsymbol{R}$ and $\boldsymbol{F}$ are the vectors of nodal external and internal forces, respectively. In the internal nodal forces $\boldsymbol{F}$ are time functions of nodal displacements $\boldsymbol{U}$ for the time interval $t+\Delta t$, the equilibrium condition needs a iterative approach. The iterative process can be described as [18]:

$$
\begin{gathered}
{ }^{t+\Delta t} \boldsymbol{U}^{(0)}={ }^{t} \boldsymbol{U} \text { and }{ }^{t+\Delta t} \boldsymbol{F}^{(0)}={ }^{t} \boldsymbol{F} \\
\Delta \boldsymbol{R}^{(k-1)}={ }^{t+\Delta t} \boldsymbol{R}-{ }^{t+\Delta t} \boldsymbol{F}^{(k-1)} \\
{ }^{t+\Delta t} \boldsymbol{K}^{(k)} \Delta \boldsymbol{U}^{(k)}=\Delta \boldsymbol{R}^{(k-1)} \\
{ }^{t+\Delta t} \boldsymbol{U}^{(k)}={ }^{t+\Delta t} \boldsymbol{U}^{(k-1)}+\Delta \boldsymbol{U}^{(k)}
\end{gathered}
$$

where $k$ is the iteration time and $\boldsymbol{K}$ is the Jacobian matrix. The equilibrium condition (9) between internal and external forces is achieved at every iteration using the Newton-Raphson method [16]. With this method, the tangential stiffness matrix is formed and decomposed at every iteration [18]. Node trajectories are used to find the HD kinematic error as the difference between the input divided by $i$, and output angles. The kinematic error analysis includes linear regression and the mean of the residuals (Section 3.2).

\subsection{Stress analysis, fatigue and pitting strengths}

The finite element dynamic analysis in this work incorporates kinematics of deformable bodies and the nonlinear effects of localized stress between internal rigid spline, external flexible spline, and wave generator. Due to the absence of stresses in the axial direction, the finite element analysis is done under plane stress. This condition is verified by two factors: (1) the reduced incidence of the rotational dynamics of the transmission components in the generation of axial forces, and (2) the absence of geometric constraints that may result in restrictions on the free strain in the axial direction. As required by isoparametric finite elements, the nodal displacement equations make use of the Jacobian transformation matrix to transform natural to Cartesian coordinates [18]. Our model ensures that the sign of the determinant of the Jacobian matrix is constant. The finite elements define their displacements according to interpolation functions, making nodes comply with the conditions imposed by the strain energy functions and requirements of convergence. Finally, the stress distribution is determined from the nodal displacements.

The non-linear finite element model of the tooth external surface includes contact nodes. The internal surface of the flexible spline and the wave generator make use of surface-to-surface contact [16]. The material model for all elements corresponds to a steel alloy AISI SAE 4340 of elastic modulus 210 GPa and elastic limit 620.4 MPa [19]. In this model, the rigid spline is fixed. The input shaft has an angular velocity of $60 \mathrm{rpm}$ with an input torque of $1.77 \mathrm{Nm}$ per millimeter in tooth face width. The number of teeth in the rigid and the flexible splines are 115 and 113, respectively. A dynamic friction coefficient of 0.07 is considered in all surfaces. Meshing control is applied to all surfaces in contact [16].

The mechanical strength analysis uses von Mises distortion energy considering maximum and minimum equivalent oscillating stresses of the double wave HD. The fatigue model uses the modified Goodman criterion [20]. Surface failure can occur due to the complex contact condition among teeth involving rolling and sliding. The pitting failure analysis considers results from experimental combining rolling and sliding [21]. Fatigue and pitting strength analysis are considered on the flexible spline, which is the most critical component in the HD transmission. The evaluation of the fatigue and pitting failure requires the location of the most probable point of failure. The location of the most probable point of failure in a double wave HD cannot be pre-established without full-system non-linear FEA. To this end, our work considers six nodes uniformly separated along the tooth profile. The time history of stress is analyzed in these six nodes. Figure 2 shows the finite element mesh and two analysis nodes. 


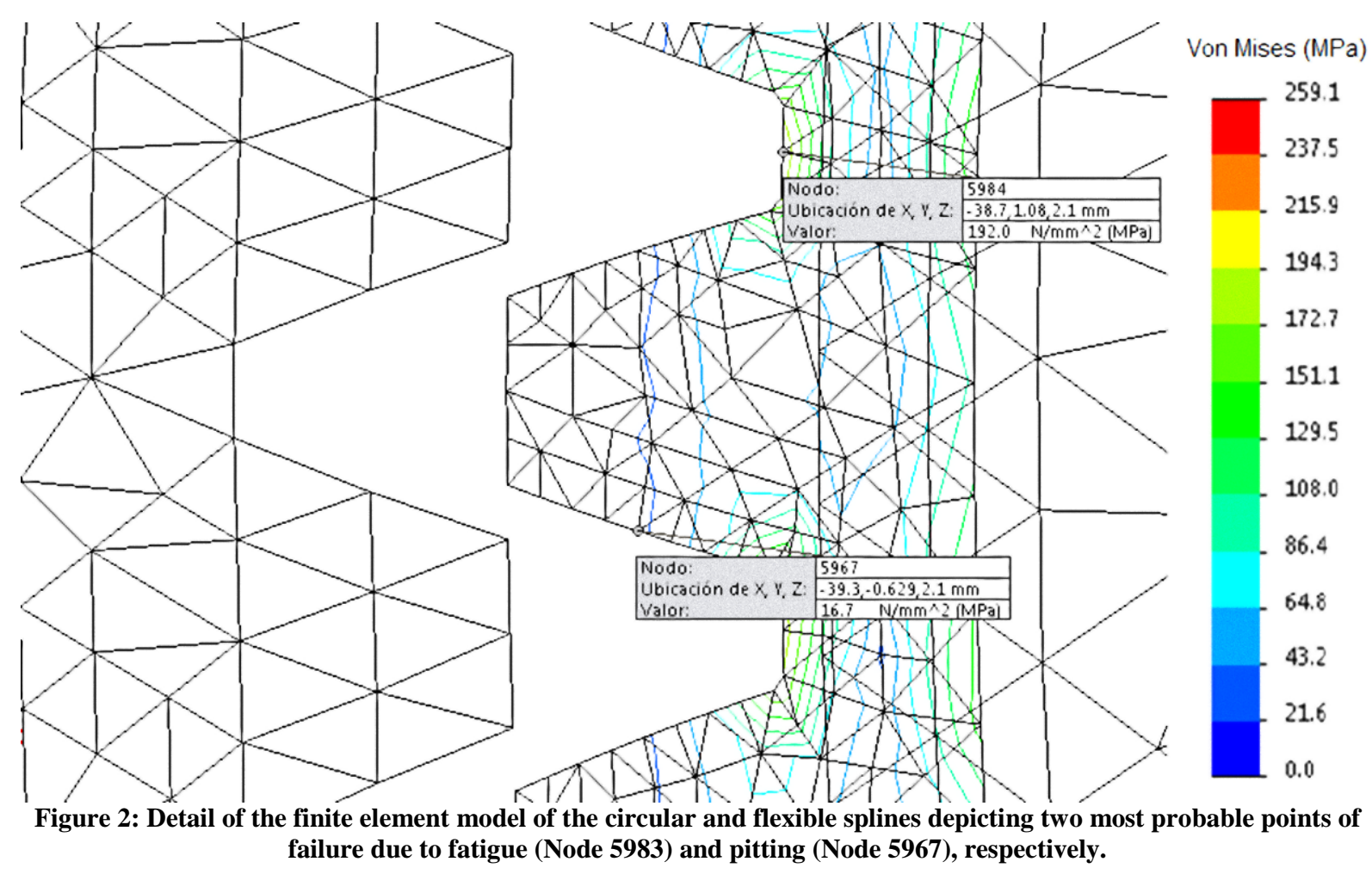

The fatigue safety factor $S_{f}$ is defined as a function of the equivalent mean value and the amplitude of the von Mises stress as [22]:

$$
S_{f}=\frac{\sigma_{e} \sigma_{u t}}{\sigma_{V M a} \sigma_{u t}+\sigma_{V M m} \sigma_{e}}
$$

where $\sigma_{e}$ is the corrected endurance limit for the flexible spline material, $\sigma_{u t}$ is the ultimate tension stress, and $\sigma_{V M m}$ and $\sigma_{V M a}$ are the equivalent mean and amplitude of the von Misses stress, respectively. The number of cycles for pitting failure $N_{p}$ is determined as [22]:

where

$$
N_{p}=10^{\vartheta-\gamma \log K}
$$

$$
K=\pi \sigma_{z}\left(m_{1}+m_{2}\right),
$$

and $\vartheta, \gamma$ are pitting strength empirical parameters, $\sigma_{z}$ is the peak normal stress on the teeth of the flexible spline, and $m_{1}$ and $m_{2}$ are material elastic properties of the rigid and flexible splines, respectively. For the HD, the empirical parameters and material elastic properties are obtained for a slip-to-roll ratio of $42.8 \%$ [22]. The input power is considered constant; however, the gear dimensions are a function of the geometric variables and they vary according to the full factorial design of experiments described in the following section.

\subsection{Design of experiments}

A three-level, full-factorial experimental design is applied in order to determine the influence of the pressure angle, modulus, and tooth correction factor on the load capacity, kinematic transmission error, and fatigue and pitting failure. Our approach considers three design factors and eight parameters (Figure 3). 


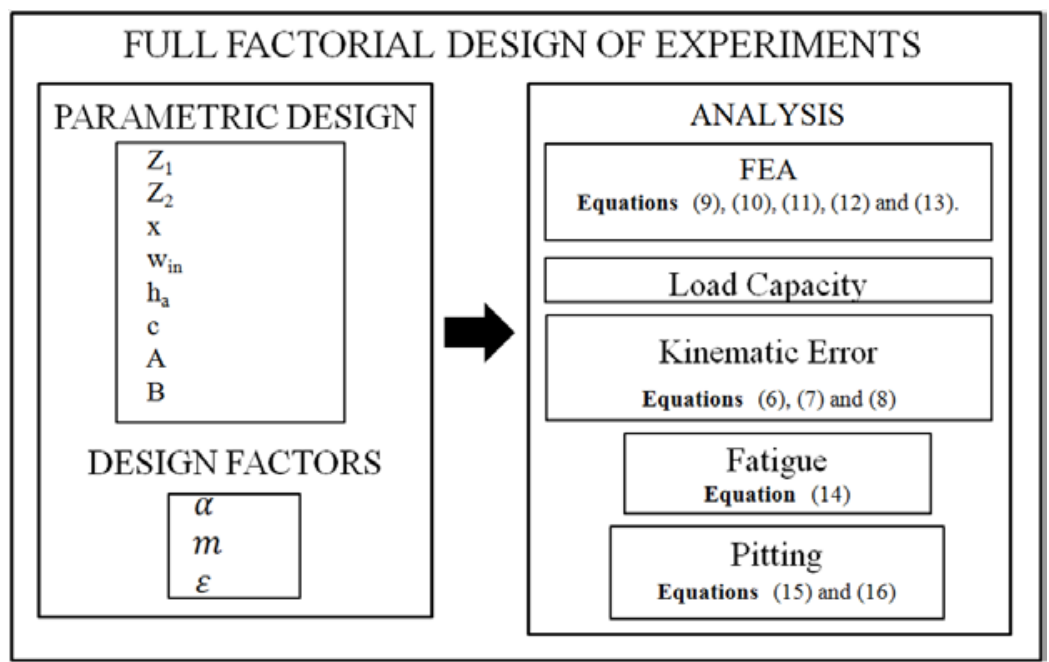

Figure 3: Full-factorial design of experiments methodology used in this research.

A systematic analysis was conducted where three geometric factors were varied according to a three level full factorial design. The factors were: gear modulus, pressure angle, and tooth correction factor. Finally, $3^{3}=27$ variants of geometric design were obtained. Table 1 shows the values used in the experiments.

Table 1: Parameters used in the full factorial design of experiments.

\begin{tabular}{|c|c|c|c|}
\hline $\mathrm{N}$ & $\begin{array}{l}\text { Gear modulus } \\
(\mathrm{mm})\end{array}$ & $\begin{array}{c}\text { Pressure } \\
\text { angle } \\
\text { (degrees) }\end{array}$ & $\begin{array}{c}\text { Tooth } \\
\text { correction } \\
\text { factor }\end{array}$ \\
\hline 1 & 0.1 & 20 & 0 \\
\hline 2 & 0.1 & 20 & 0.25 \\
\hline 3 & 0.1 & 20 & 0.50 \\
\hline 4 & 0.1 & 25 & 0 \\
\hline 5 & 0.1 & 25 & 0.25 \\
\hline 6 & 0.1 & 25 & 0.50 \\
\hline 7 & 0.1 & 30 & 0 \\
\hline 8 & 0.1 & 30 & 0.25 \\
\hline 9 & 0.1 & 30 & 0.50 \\
\hline 10 & 0.4 & 20 & 0 \\
\hline 11 & 0.4 & 20 & 0.25 \\
\hline 12 & 0.4 & 20 & 0.50 \\
\hline 13 & 0.4 & 25 & 0 \\
\hline 14 & 0.4 & 25 & 0.25 \\
\hline 15 & 0.4 & 25 & 0.50 \\
\hline 16 & 0.4 & 30 & 0 \\
\hline 17 & 0.4 & 30 & 0.25 \\
\hline 18 & 0.4 & 30 & 0.50 \\
\hline 19 & 0.7 & 20 & 0 \\
\hline 20 & 0.7 & 20 & 0.25 \\
\hline 21 & 0.7 & 20 & 0.50 \\
\hline 22 & 0.7 & 25 & 0 \\
\hline 23 & 0.7 & 25 & 0.25 \\
\hline 24 & 0.7 & 25 & 0.50 \\
\hline 25 & 0.7 & 30 & 0 \\
\hline 26 & 0.7 & 30 & 0.25 \\
\hline 27 & 0.7 & 30 & 0.50 \\
\hline
\end{tabular}


An analysis of the results of the full factorial design using a statistical analysis of variance (ANOVA) was performed. The response surfaces and empirical models for the kinematic error, load capacity, bending fatigue strength, and pitting were obtained using statistical analysis system software. The total correction factor in both splines satisfies that [23]:

$$
\xi_{1}+\xi_{2}=0
$$

and for the externally geared flexible spline the correction factors satisfies that [15]:

$$
\xi_{2} \geq 0
$$

The results of the analysis of invariance are presented in the Appendix and analyzed in the following section.

\subsection{Load capacity}

\section{Results and Discussion}

The theoretical geometric model depicts interference so the addendum in all teeth had to be reduced in proportion similar to the variation of all geometric parameters. Considering all deflections, a reduction of $22 \%$ in the height of both gearing teeth allows a full cycle without compromising the tooth strength. With a reduction of $30 \%$ the performance characteristics are decreased, but they still fall in a range of what can be considered a normal operation. The effect of the geometry on the load capacity is statically determined. The correction factor conditions (17) and (18) decreases the transversal section area of the splines, increasing the load capacity. Figure 4 shows the effect of the pressure angle, modulus, and correction factor on the load capacity. The maximum load capacity of $5.86 \mathrm{~kW} / \mathrm{kg}$ corresponds to a pressure angle of $20^{\circ}$, modulus 0.1 and tooth correction factor 0.50 . While the load capacity is highly influenced by the gear modulus, it is practically insensitive to the pressure angle and the correction for the ranges considered.

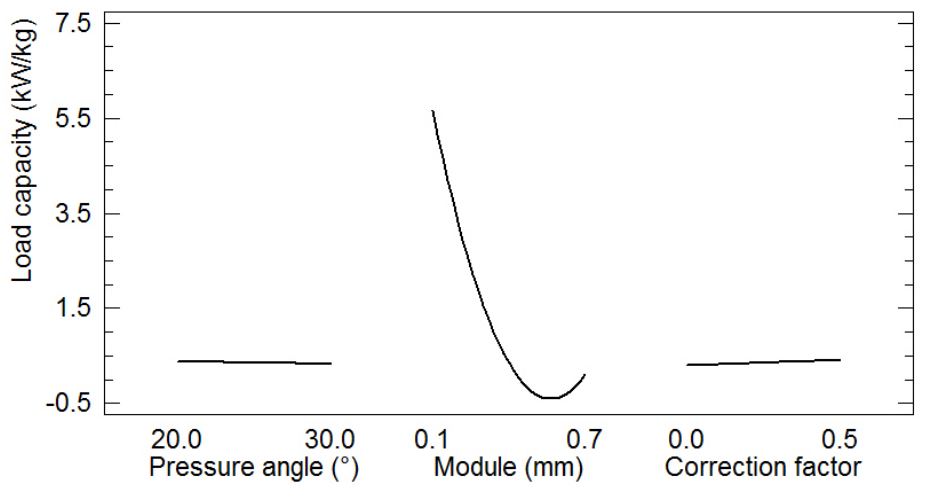

Figure 4: Effect of the pressure angle, modulus, and correction factor on the HD load capacity.

\subsection{Kinematic error analysis}

Two finite element models are developed for the kinematic numerical analysis of the HD: without and with teeth (Figure 5). In both cases the thickness of ring is the same. For the same power efficiency, the maximum von Mises stress results for the toothless case is lower than the one corresponding to the more realistic and numerically more expensive case with teeth. In both cases, the relative stress distribution is very close. 


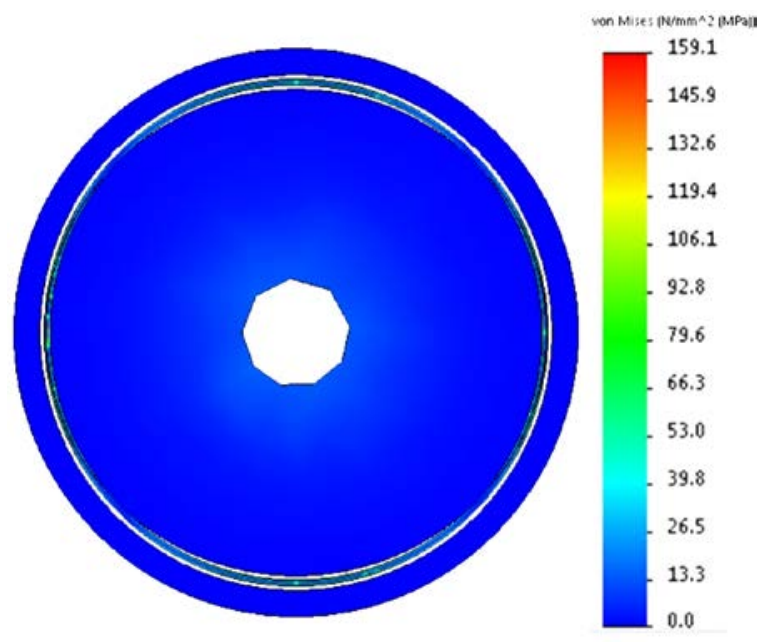

(a)

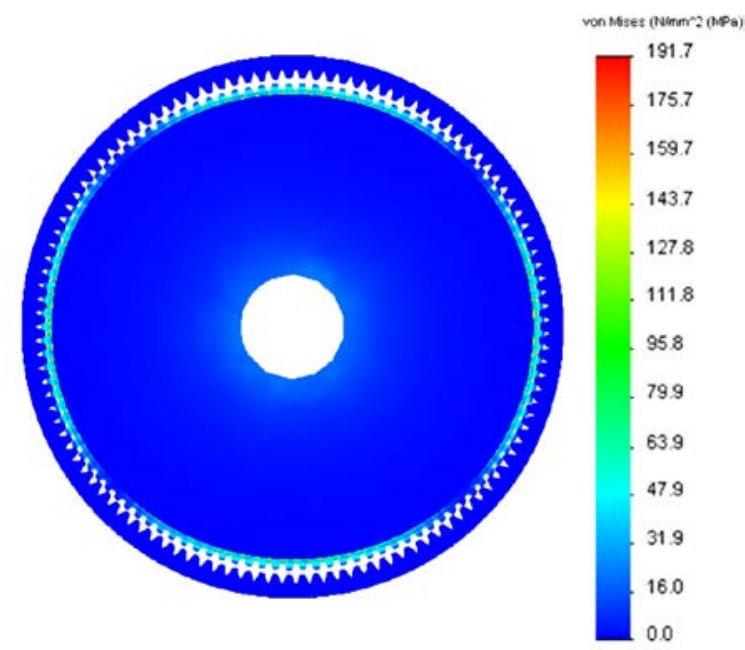

(b)

Figure 5: Finite element-based kinematic models of the HD: (a) without teeth, and (b) with teeth.

The simulation of trajectory of a point on the surface of the flexible spline depicts differences depending on the model. Figure 6 shows the trajectory in global rectangular coordinates for the analytical and numerical, finite element-based models (with and without teeth) in three $360^{\circ}$ cycles. While the analytic model and the teethed finite element models predict similar motion ranges, the toothless model shows a significant delay corresponding to $\sim 1^{\circ}$ after the third cycle. However, the wave transmission of both numerical models is close in shape for which both are equivalent predictions with different kinematic gain.

A linear regression adjustment is used to determine the transmission error residuals. Figure 7 shows the error residuals in the output shaft for the double wave HD with pressure angle $20^{\circ}$, modulus $0.1 \mathrm{~mm}$, and correction factor +0.50 . The mean residual is the linear fit of the numerical results. The range spanned between the maximum and minimum values defines the kinematic error of the transmission.

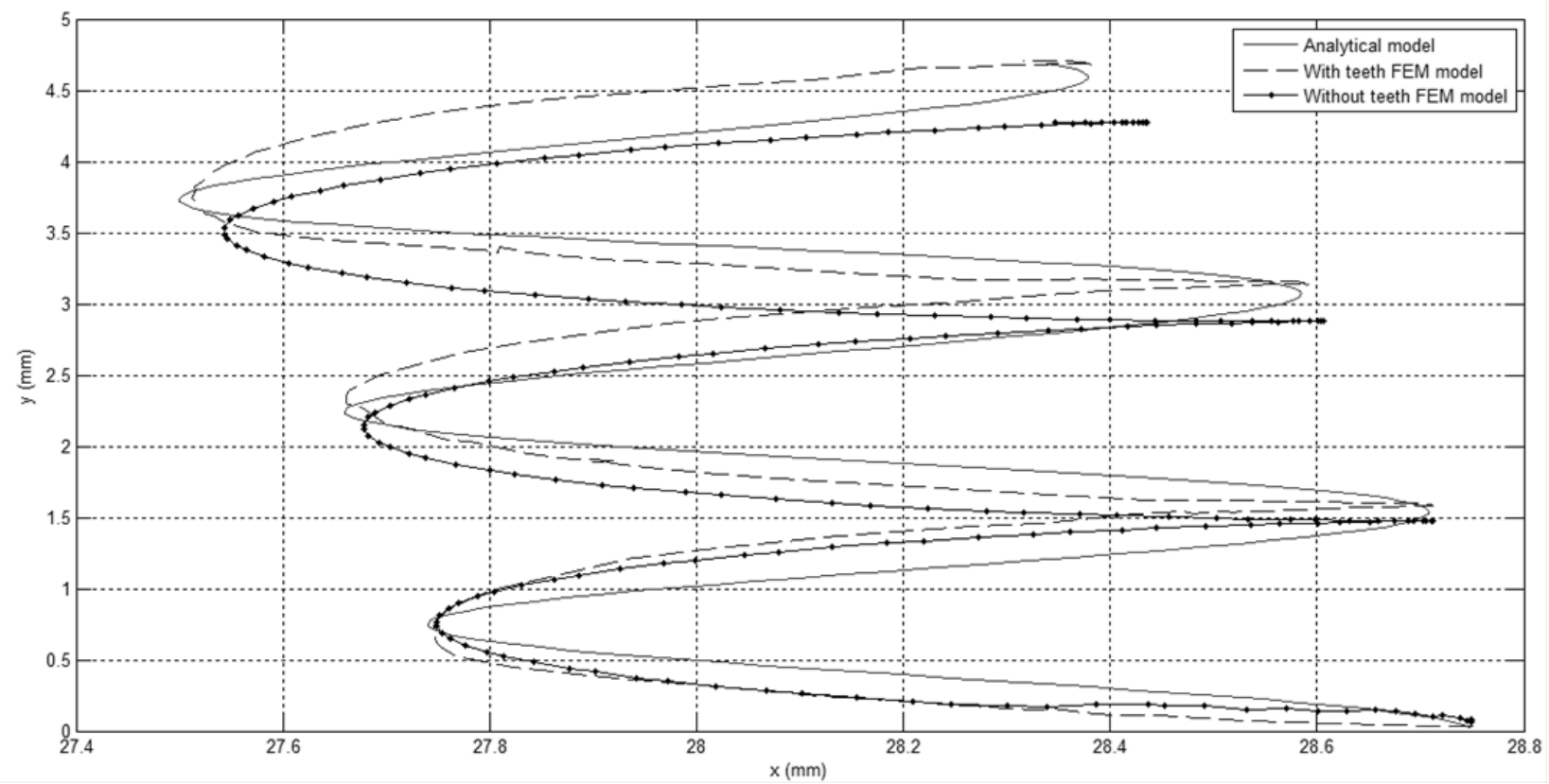

Figure 6: Trajectory described by a point on the surface of the flexible spline described by the analytical model, and the finite element models with and without teeth. 


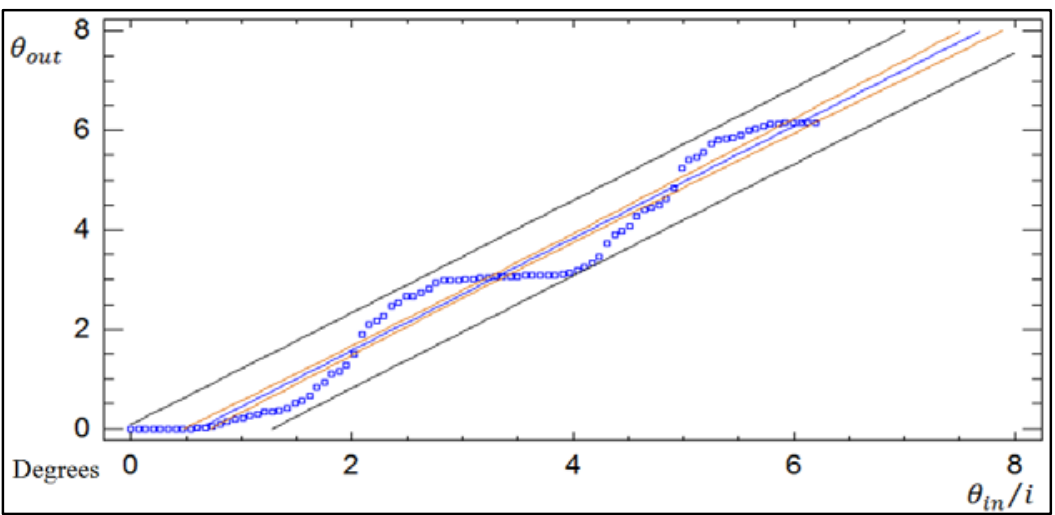

Figure 7: Linear regression adjustment used to determine the kinematic transmission error (units in degrees).

Statistical analysis is used to determine the influence of the pressure angle, modulus, and correction factor on the HD average transmission error (Figure 8). Appendix contains the detailed stochastic analysis. A smaller transmission error is obtained for a larger correction factor and a smaller pressure angle. For these conditions, the trajectory of the point of contact in the flexible spline tends to be more linear decreasing the variation between the input-to-output ratio and, therefore, the transmission kinematic error. A minimum transmission error of $0.274^{\circ}$ is obtained for pressure angle $20^{\circ}$, modulus $0.10 \mathrm{~mm}$, and correction factor +0.5 .

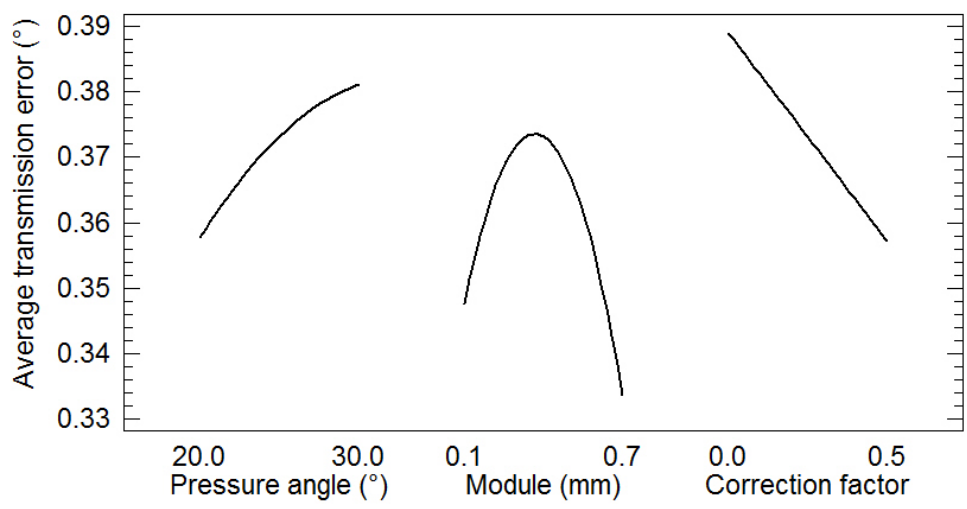

Figure 8: Effect of the pressure angle, modulus, and correction factor on the average kinematic transmission error.

\subsection{Bending fatigue and pitting}

Time varying stress and strain conditions are determined from the dynamic finite element analysis. This analysis shows that the flexible spline depicts higher normal contact Hertz stress on the tooth surface and von Misses stress on the tooth base as a product of the strains induced by the wave generator and the resulting gearing. Figure 9 shows the gearing position between the rigid and flexible splines for which highest stresses are obtained.

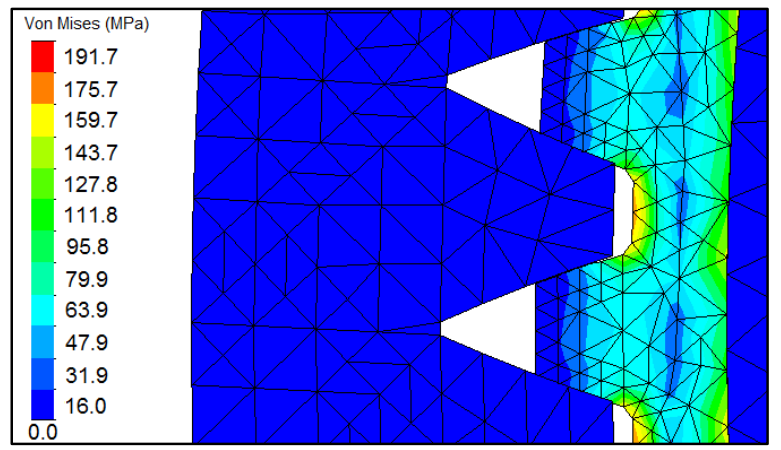

Figure 9: Gear position for maximum surface normal Hertz contact stress and corresponding maximum effective von Mises stress in the tooth base. 
Figure 10 shows the variation of the surface normal Hertz contact stress on the tooth surface and the von Mises stress on the tooth base for a full $360^{\circ}$ rotation of the input shaft. This simulation shows corresponding peak and valley locations for both curves. The contact stress values are used by the pitting strength analysis.

Bending fatigue and pitting analysis make use of (14), (15) and (16). Appendix contained the detailed stochastic analysis for fatigue strength and pitting, respectively. This analysis shows the influence of the pressure angle, modulus, and correction factor on the fatigue factor of safety $S_{f}$ on the tooth base. Figure 11 shows the effect of the pressure angle, modulus, and correction factor on the fatigue safety factor on the tooth base. The highest value of $S_{f}=2.57$ corresponds to pressure angle $21.7^{\circ}$, modulus 0.55 , and tooth correction factor +0.50 . The bending fatigue strength is monotonically increased with respect to the tooth correction factor due to the increment on the tooth base area. The pressure angle and modulus have a larger influence on the tooth profile for which favoring the bending stress on the tooth base. Finally, all 27 models showed excellent pitting strength; however, the maximum value is obtained for pressure angle $27^{\circ}$, modulus 0.7 , and correction factor 0.0 .

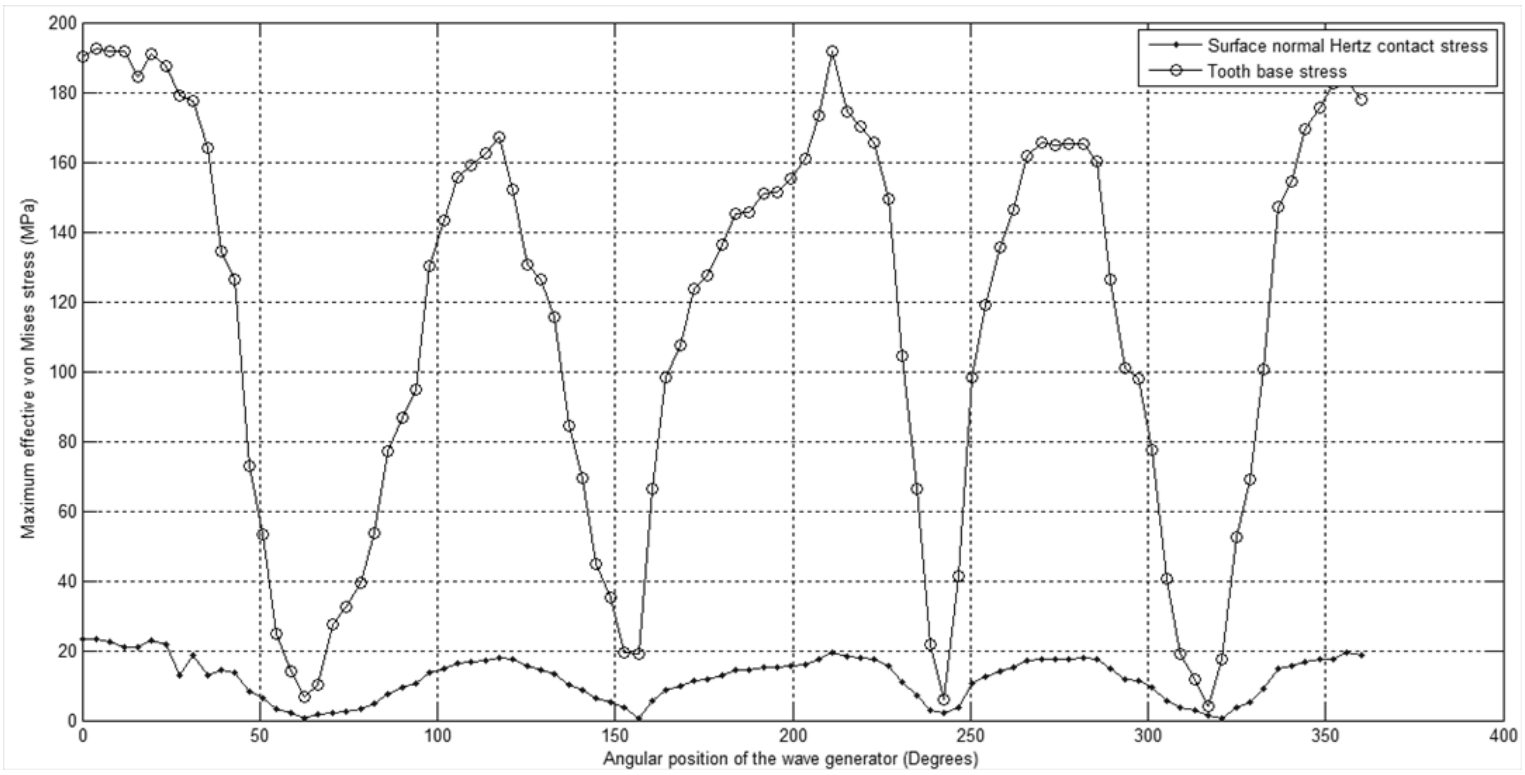

Figure 10: Cyclic variation of the surface normal Hertz contact stress and corresponding maximum effective von Mises stress in the tooth base as a function of the angular position of the wave generator.

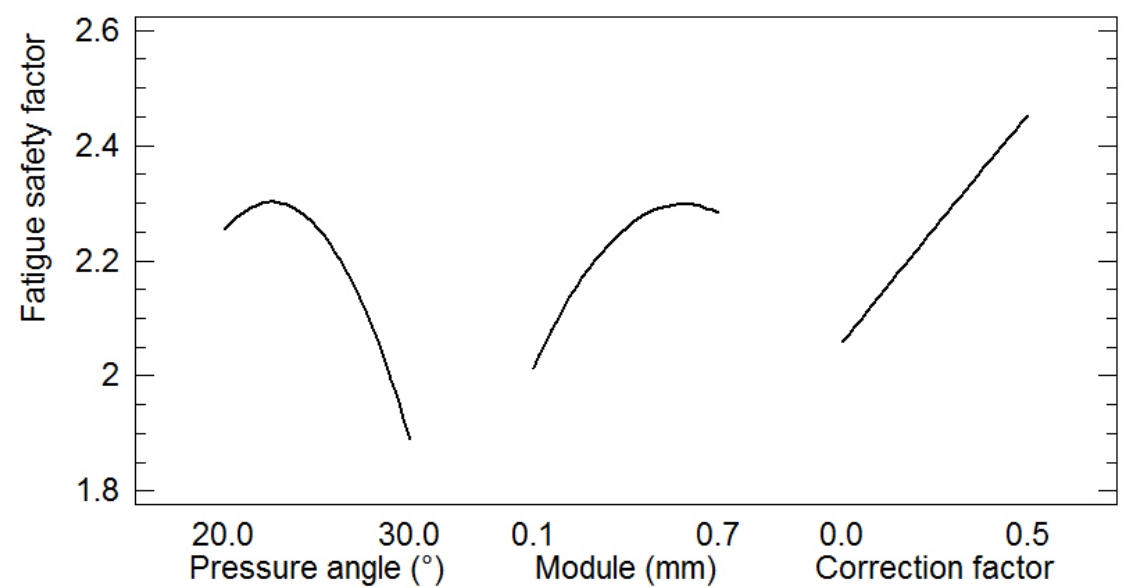

Figure 11: Effect of the pressure angle, modulus, and correction factor on the fatigue safety factor on the tooth base.

\section{Final remarks}

This work introduces a systematic approach for harmonic drive design considering nonlinear dynamic analysis, particularly cycling contact stresses between the three main components of the transmission (rigid spline, flexible 
spline, and wave generator) and stresses at the teeth root. Analytic and numerical finite element-based models are utilized on a double wave HD. These models allowed to measuring the influence of pressure angle (from $20^{\circ}$ to $30^{\circ}$ ), modulus (from 0.1 to $0.7 \mathrm{~mm}$ ), and tooth correction factor (from 0.0 to +0.5 ) on load capacity, kinematic transmission error, and fatigue and pitting strengths. Three-level full factorial experimental design supports the statistical analysis of this work. Even though, Taguchi planning or general reduced fractional factorial design is computationally efficient due to the reduced number of simulations, our work supports the more computational expensive full-factorial study to quantify the effect of design variables in the non-linear HD performance. A noise in the planning is also an alternative, but its use would make it more difficult to accurately reproduce the results; therefore, deterministic design of experiments was followed in this work.

The finite element models include a full-size, fully toothed harmonic drive, which is not commonly presented by numerical studies. The results obtained with this model are compared to the ones from the more commonly used toothless model as well as the results from the analytic equations. The transmission errors predicted by the model with teeth agree more closely to the results from the analytic model than to the results from the toothless numeric model. The toothed numerical model also allows to determining and correcting interference problems that cannot be detected by any other modeling approach.

Load capacity studies performed in this paper shows the main influence on the modulus. In particular, this work shows a dramatic load capacity improvement when the modulus is decreased to order of $0.1 \mathrm{~mm}$. However, the low modulus value increases the manufacturing cost due to the required special tooling. Interestingly, load capacity is not sensitive to changes in pressure angle or correction factor. A load capacity larger than $5.8 \mathrm{~kW} / \mathrm{kg}$ is theoretically achievable in a well-designed harmonic drive.

Kinematic transmission error is significantly influenced by all parameters considered and is generally favored by lower pressure angle, higher modulus, and higher correction factor. These results can be explained from the wave uniformity at this regime. A kinematic error of less than $0.3^{\circ}$ is theoretically achievable with the correct harmonic drive design.

Pitting occurs after a very long period of time and this study shows that it will not be a concern for the harmonic drive. The reason is because the contact stresses on the tooth are relatively smaller than the bending stresses on the tooth base. Bending fatigue is clearly influenced by the design parameters. In all cases von Mises stresses (mean and amplitude) fall in a safe operation regime according the modified Goodman's fatigue failure criterion. A safety factor larger than 2.5 is theoretically possible to obtained with the proper harmonic drive design.

Future work includes the development of a 3D model employing a dynamic finite element analysis of nonlinear isotropic hyperelastic material for the splines. This type of model is more appropriate to describe certain polymers which may be potentially useful in this type of transmission. Furthermore, it is necessary to conduct a detailed study on the influence that has the tooth addendum height in the HD performance. This geometric parameter should be included in future virtual experimental design.

\section{Acknowledgments}

The National University of Colombia at Bogotá and the Indiana Space Grant Consortium (INSGC) supported this research effort. Any opinions, findings, conclusions, and recommendations expressed in this paper are those of the writers and do not necessarily reflect the views of the sponsors.

\section{Appendix - Fitted models}

\subsection{Load capacity}

The equation for the fitted model is:

$$
\begin{gathered}
L C=8.77151-0.0160725 \phi-32.0864 m+0.672273 \xi+0.0000453607 \phi^{2}+0.0224681 \phi m \\
-0.00063109 \phi \xi+28.1708 m^{2}-1.03049 m \xi-0.0327807 \xi^{2}
\end{gathered}
$$

with $\mathrm{R}^{2}=99.98 \%$, mean absolute error $=0.0241476$, and Durbin-Watson statistic $=1.21314(\mathrm{P}=0.0031)$, confidence interval LC $=[3.57501 ; 5.99652]$. The response surface is: 


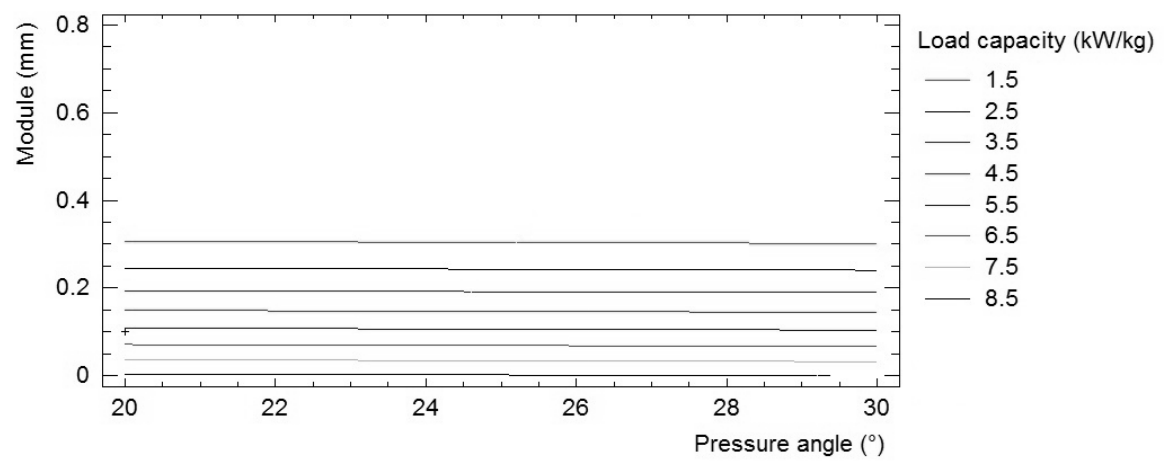

Figure 12: Load capacity response for a correction factor $\xi=0.25$ as a function of $m$ and $\Phi$.

\subsection{Kinematic transmission error}

The equation for the fitted model is:

$$
\begin{aligned}
\theta_{e}=0.294959+ & 0.00563235 \phi+0.165081 m-0.3339 \xi-0.000149184 \phi^{2}+0.00381561 \phi m \\
& +0.0105043 \phi \xi-0.361116 m^{2}+0.0211167 m \xi-0.00106844 \xi^{2}
\end{aligned}
$$

with $\mathrm{R}^{2}=24.33 \%$, mean absolute error $=0.0352834$, and Durbin-Watson statistic $=1.61063(\mathrm{P}=0.0426)$, confidence interval $\theta_{\mathrm{e}}=[0.30854 ; 0.38938]$. The response surfaces are:

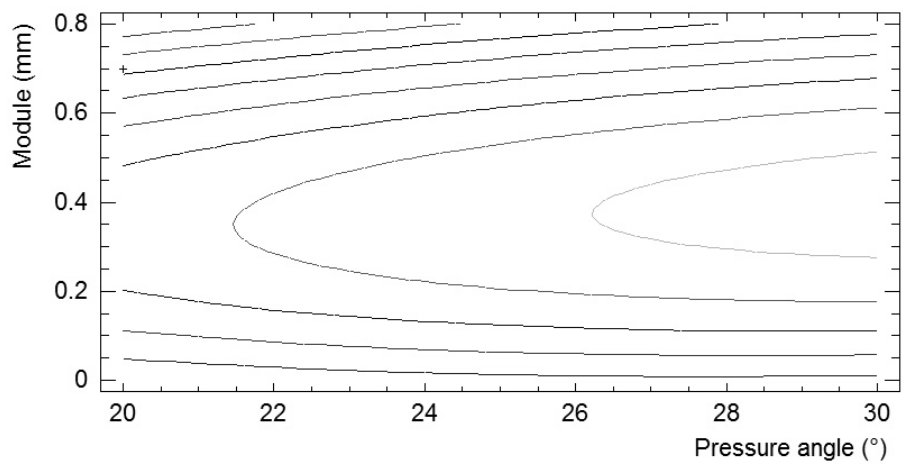

Kinematic transmission error $\left({ }^{\circ}\right)$

0.292

0.304

0.316

0.328

0.34

0.352

0.364

0.376

Figure 13: Kinematic transmission error for a correction factor $\xi=0.25$ as a function of the $m$ and $\Phi$.

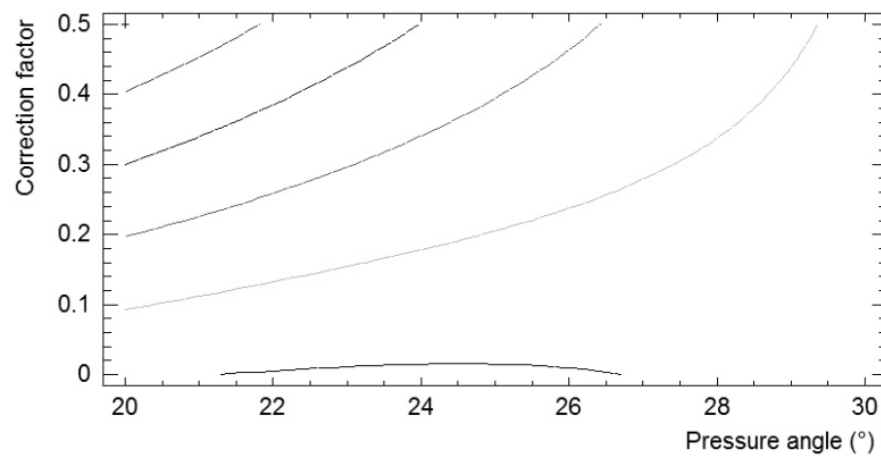

Kinematic transmission error $\left({ }^{\circ}\right)$

0.34

0.352

0.364

0.376

$-0.388$

Figure 14: Kinematic transmission error for a modulus $\boldsymbol{m}=\mathbf{0 . 4 0} \mathrm{mm}$ as a function of $\xi$ and $\Phi$.

\subsection{Fatigue strength}

The equation for the fitted model is:

$$
\begin{aligned}
S_{f}=-2.23008+ & 0.34134 \phi+0.960787 m+2.35171 \xi-0.007408 \phi^{2}+0.0194722 \phi m-0.05992 \phi \xi \\
& -1.21444 m^{2}-0.117778 m \xi-0.0370667 \xi^{2}
\end{aligned}
$$

with $\mathrm{R}^{2}=69.95 \%$, mean absolute error $=0.135503$, Durbin-Watson statistic $=2.60236(\mathrm{P}=0.7453)$, confidence interval $S_{f}=[1.77552 ; 2.34368]$. The response surfaces are: 


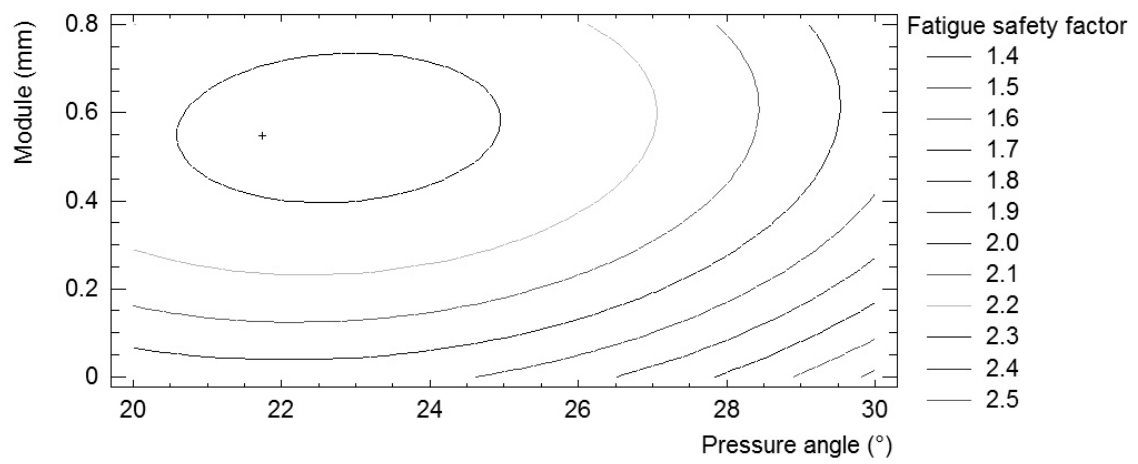

Figure 15: Fatigue safety factor for a correction factor $\xi=0.25$ as a function of the $\boldsymbol{m}$ and $\Phi$.

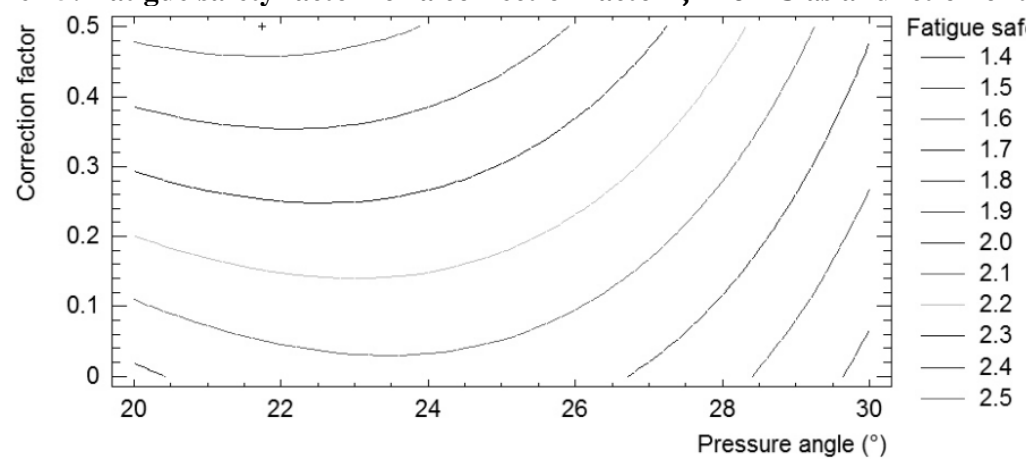

Figure 16: Fatigue safety factor for a modulus $m=0.40 \mathrm{~mm}$ as a function of $\xi$ and $\Phi$.

\subsection{Pitting life}

The equation for the fitted model is:

$$
\begin{gathered}
N_{p}=-5.5651 \mathrm{E} 91+4.5532 \mathrm{E} 90 \phi-6.3185 \mathrm{E} 89 m-4.5535 \mathrm{E} 90 \xi-9.1065 \mathrm{E} 88 \phi^{2}+9.1499 \mathrm{E} 82 \phi m \\
-1.098 \mathrm{E} 83 \phi \xi+1.2647 \mathrm{E} 91 m^{2}-2.2766 \mathrm{E} 91 m \xi+1.8213 \mathrm{E} 91 \xi^{2}
\end{gathered}
$$

with $\mathrm{R}^{2}=31.73 \%$, mean absolute error $=1.954 \mathrm{E} 90$, Durbin-Watson statistic $=2.94603(\mathrm{P}=0.9418)$, confidence interval $N_{p}=$ [2.33704E90; 3.85479E90]. The response surfaces are:

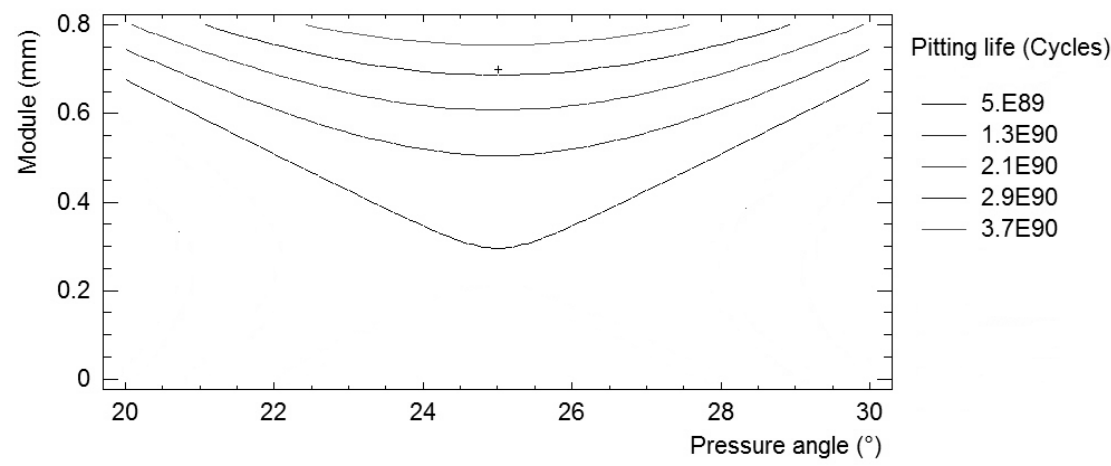

Figure 17: Pitting life for a correction factor $\xi=0.25$ as a function of the modulus and the pressure angle. 


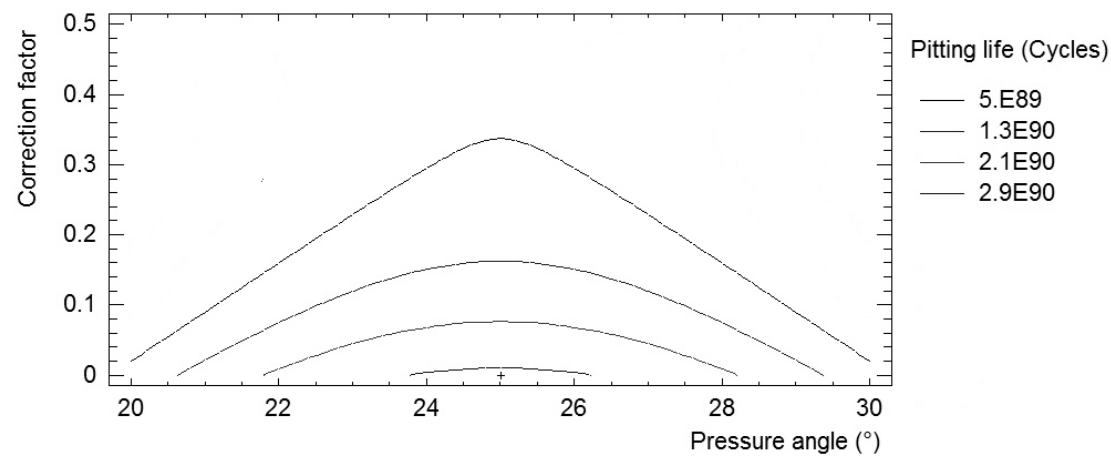

Figure 18: Pitting life for a modulus $\boldsymbol{m}=\mathbf{0 . 4 0} \mathbf{~ m m}$ as a function of the correction factor and the pressure angle.

\section{References}

1. Gervini, V.I., S.C.P. Gomes, and V.S. Da Rosa, A New Robotic Drive Joint Friction Compensation Mechanism Using Neural Networks. Journal of the Brazilian Society of Mechanical Sciences and Engineering, 2003. 25(2): p. 129-139.

2. De Lucena, S.E., M.A. Marcelino, and F.J. Grandinetti, Low-cost PWM speed controller for an electric mini-baja type vehicle. Journal of the Brazilian Society of Mechanical Sciences and Engineering, 2007. 29(1): p. 21-25.

3. Jeon, H.S. and S.H. Oh. A study on stress and vibration analysis of a steel and hybrid flexspline for harmonic drive. in 10th International Conference on Composite Structures, November 15, 1999 - November 16, 1999. 1999. Melbourne, Aust: Elsevier Ltd.

4. Kayabasi, O. and F. Erzincanli, Shape optimization of tooth profile of a flexspline for a harmonic drive by finite element modelling. Materials and Design, 2007. 28(2): p. 441-447.

5. Ostapski, W., Analysis of the stress state in the harmonic drive enerator-flexspline system in relation to selected structural arameters and manufacturing deviations. Bulletin of the Polish Academy of Sciences: Technical Sciences, 2010. 58(4): p. 683-698.

6. Gandhi, P.S. and F. Ghorbel, High-speed precision tracking with harmonic drive systems using integral manifold control design. International Journal of Control, 2005. 78(2): p. 112-121.

7. Tuttle, T.D. and W.P. Seering, Nonlinear model of a harmonic drive gear transmission. IEEE Transactions on Robotics and Automation, 1996. 12(3): p. 368-374.

8. Ostapski, W. and I. Mukha, Stress state analysis of harmonic drive elements by FEM. Bulletin of the Polish Academy of Sciences: Technical Sciences, 2007. 55(1): p. 115-123.

9. Péter, J. and G. Németh, Results of laboratory tests of harmonic gear drive. Design of Machines and Structures, 2012. 2(1): p. 35-51.

10. Dong, H. and D. Wang. Elastic deformation characteristic of the flexspline in harmonic drive. in 2009 ASME/IFToMM International Conference on Reconfigurable Mechanisms and Robots, ReMAR 2009, June 22, 2009 - June 24, 2009. 2009. London, United kingdom: IEEE Computer Society.

11. Tjahjowidodo, T., F. Al-Bender, and H. Van Brussel, Theoretical modelling and experimental identification of nonlinear torsional behaviour in harmonic drives. Mechatronics, 2013. 23(5): p. 497-504.

12. Sobieszanski-Sobieski, J., J.F. Barthelemy, and K.M. Riley, Sensitivity of optimum solutions to problem parameters. Collection of Technical Papers - AIAA/ASME/ASCE/AHS/ASC Structures, Structural Dynamics and Materials Conference, 1981(Pt 1): p. 184-205.

13. Mastinu, G., M. Gobbi, and C. Miano, Optimal Design of Complex Mechanical Systems. 2010: Springer. 359.

14. Sadler, W.C., Kinematics and Dynamics of Machinery. 3rd ed. 2003: Pearson Education.

15. González Rey, G., P. Frechilla Fernández, and R. José García Martín, Coeficiente de corrección en engranajes cilíndricos como factor de conversión entre sistemas AGMA e ISO. Ingeniería Mecánica, 2007. 10(3): p. 63-69.

16. SolidWorks, Simulation premium: Nonlinear handbook. 2010: Massachusetts, USA. 
17. Córdoba, E., et al., Transmisión flexondulatoria armónica. 2011, Bogotá, Colombia: Universidad Nacional de Colombia.

18. Bathe, K.J., Finite Element Procedures. 2003.

19. International, A., Metal Handbook. 2nd ed. 1998, USA: Editorial Advisory Board.

20. Collins, J., H. Busby, and S. G., Mechanical design of machine elements and machines - a failure prevention perspective. 2nd ed. 2009, USA.

21. T., S. and W. W., Mechanical failure: definition of the problem. Vol. NBS special publication 423. 1974, Washington D.C., USA: National Bureau of Standards.

22. Norton, R., Machine design, an integrated approach. 5th ed. 2010, USA: Prentice-Hall Inc.

23. Quinones, A., et al. Influence of the friction force, the tooth correction coefficient and the normal force radial component in the form factor and the stress in the feet of spur gear's teeth. in Proceedings of the ASME Design Engineering Division 2005, November 5, 2005 - November 11, 2005. 2005. Orlando, FL, United states: American Society of Mechanical Engineers. 